\title{
Capillary Waves at Liquid/Vapor Interfaces: A Molecular Dynamics Simulation
}

\author{
Scott W. Sides, ${ }^{\dagger}$ Gary S. Grest, ${ }^{\dagger}$ and Martin-D. Lacasse ${ }^{\ddagger}$ \\ ${ }^{\dagger}$ Sandia National Laboratories, Albuquerque, New Mexico 87185-1411 \\ ${ }^{\ddagger}$ Corporate Research Science Laboratories, Exxon Research and Engineering Company, Annandale, New Jersey 08801
}

(June 28, 1999)

\begin{abstract}
Evidence for capillary waves at a liquid/vapor interface are presented from extensive molecular dynamics simulations of a system containing up to 1.24 million Lennard-Jones particles. Careful measurements show that the total interfacial width depends logarithmically on $L_{\|}$, the length of the simulation cell parallel to the interface, as predicted theoretically. The strength of the divergence of the interfacial width on $L_{\|}$depends inversely on the surface tension $\gamma$. This allows us to measure $\gamma$ two ways since $\gamma$ can also be obtained from the difference in the pressure parallel and perpendicular to the interface. These two independent measures of $\gamma$ agree provided that the interfacial order parameter profile is fit to an error function and not a hyperbolic tangent, as often assumed. We explore why these two common fitting functions give different results for $\gamma$.
\end{abstract}

PACS number(s): 68.35.Ja 68.35.Md 64.70.Fx 68.35.Ct 68.10.-m

An interface is the physical boundary between two distinct thermodynamic phases, i.e. a region characterized by a local gradient of the order-parameter which mean value changes from one phase to the other. Examples include domain boundaries in ferromagnetic materials, the interface between two immiscible liquids, or between a liquid and its own vapor below the critical temperature $T_{c}$. This last case has been well studied, both theoretically and experimentally. For simple fluids interacting via van der Waals forces, the mean local density changes monotonically [1] across the interface from its bulk liquid value to that of the vapor. In other systems, such as alkali metals for example [2,3], the profile across the interface is often more complex, with oscillations in the local density superimposed on the decaying density profile.

For simple fluids, thermodynamic considerations alone would predict that the interfacial width $w$, depends only on temperature and on the interaction energies within each phase and across the interface. However, the presence of the interface breaks the translational invariance of the system, inducing Goldstone fluctuations or "capillary waves" at an interface $[4,5]$. For two-dimensional interfaces, these non-critical fluctuations give rise to a logarithmic increase in the interfacial width $w$ with increasing $L_{\|}$, the length of the interface. Evidence for capillary waves has been found experimentally from X-ray scattering [6-8] on liquid/vapor interfaces and neutron reflectivity [9-11] on polymer/polymer interfaces. Moreover, nuclear reaction analysis (NRA) depth profiling [12] has been used to directly investigate the film thickness dependence on the interface width between two polymer films and is in qualitative agreement with capillary-wave predictions. Capillary waves have also also been observed in computer simulations for polymer/polymer interfaces [12-16]. Most previous simulations $[17,18]$ of the liquid/vapor interface in three dimensions did not investigate the dependence of $w$ on the size of the interface. One recent simulation study [19] of a thin polymer-film system gave some evidence for capillary waves, but the longitudinal size of the interface was very small.

The purpose of this paper is to present computer simulation results of interfaces in a liquid/vapor system. To our knowledge, these simulations are the most extensive studies of the interface fluctuations due to capillary waves. In particular, we obtain the surface tension $\gamma$ two different ways: from the dependence of $w$ on $L_{\|}\left(\gamma_{w}\right)$, and from the difference in pressure parallel $p_{\|}$and perpendicular $p_{\perp}$ to the interface $\left(\gamma_{p}\right)$. We find the surprising result, that $\gamma_{w}$ depends on the functional form chosen to fit the order parameter profile through the interface. In particular, fitting the profile to an error function gives results for $\gamma_{w}$ which are in excellent agreement with $\gamma_{p}$. However, fitting our data to $\tanh (2 z / w)$, a functional form derived from mean-field arguments [5], gives results for $\gamma_{w}$ which are systematically $15 \%$ smaller than $\gamma_{p}$. Since the tanh function is often used to fit interfacial profiles at the liquid/vapor interface $[13,19]$, this difference is important to understand.

For this study we perform continuous-space, molecular dynamics simulations on a system of particles interacting through a standard (12-6) Lennard-Jones potential. The potential between particles $i$ and $j$ takes the form

$$
U\left(r_{i j}\right)= \begin{cases}4 \epsilon\left[\left(\frac{\sigma}{r_{i j}}\right)^{12}-\left(\frac{\sigma}{r_{i j}}\right)^{6}\right] & r_{i j}<r_{c}, \\ 0 & r_{i j}>r_{c}\end{cases}
$$

where $r_{i j}$ is the distance between particles $i$ and $j$, and $\epsilon$ and $\sigma$ set the energy and length scales of the potential respectively. Here we take a cut-off of $r_{c}=2.5 \sigma$. Increasing $r_{c}$ merely shifts $T_{c}$ to higher values, which should have little effect on the capillary-wave properties while increasing computation time significantly. The trajectories of the $N$ particles of mass $m$, are obtained by stepwise integration of Newton's equations of motion (EOM)

$$
m \frac{d^{2} r}{d t^{2}}=-\nabla U(r)-m \Gamma \frac{d r}{d t}+W(t) .
$$




\section{DISCLAIMER}

This report was prepared as an account of work sponsored by an agency of the United States Government. Neither the United States Government nor any agency thereof, nor any of their employees, make any warranty, express or implied, or assumes any legal liability or responsibility for the accuracy, completeness, or usefulness of any information, apparatus, product, or process disclosed, or represents that its use would not infringe privately owned rights. Reference herein to any specific commercial product, process, or service by trade name, trademark, manufacturer, or otherwise does not necessarily constitute or imply its endorsement, recommendation, or favoring by the United States Government or any agency thereof. The views and opinions of authors expressed herein do not necessarily state or reflect those of the United States Government or any agency thereof. 


\section{DISCLAIMER}

Portions of this document may be illegible in electronic image products. Images are produced from the best available original document. 


\begin{tabular}{c|cccc}
\hline \hline$T\left[\epsilon / k_{\mathrm{B}}\right]$ & $N$ & $L[\sigma]$ & $L_{\perp}[\sigma]$ & time $[\tau]$ \\
\hline \hline 0.8 & 7200 & 12.8 & 127.0 & 6000 \\
& 24000 & 24.7 & 127.0 & 6000 \\
& 69360 & 42.0 & 127.0 & 6000 \\
& 154400 & 54.5 & 195.6 & 5000 \\
& 506880 & 94.0 & 125.6 & 2800 \\
\hline 0.9 & 14400 & 15.1 & 216.1 & 11000 \\
& 40000 & 25.2 & 216.1 & 10000 \\
& 115660 & 42.9 & 216.1 & 7800 \\
& 154400 & 54.5 & 195.6 & 5800 \\
& 506880 & 94.0 & 144.4 & 4200 \\
& 1240000 & 134.6 & 164.4 & 4200 \\
\hline 1.0 & 14400 & 13.3 & 264.2 & 16500 \\
& 48000 & 25.7 & 264.2 & 14400 \\
& 138720 & 43.7 & 264.2 & 10500 \\
& 170000 & 54.5 & 195.6 & 13500 \\
& 590000 & 94.0 & 293.9 & 4900 \\
\hline
\end{tabular}

TABLE I. Values used for the parameters of simulations: temperature $T$, number of particles $N, L=L_{\|}, L_{\perp}$, and duration of run.

In addition to the force derived from the LJ potential, the EOM contains a velocity-dependent damping term and a noise term representing a viscous drag force and a weak stochastic force, respectively. The noise term $W(t)$ is taken from a uniform distribution, which mean value is set from the temperature $T$ and the damping coefficient $\Gamma$ through the fluctuation-dissipation theorem [20]. The combination of the viscous damping and stochastic force terms in the EOM effectively couples the system to a heat bath. Our simulations are performed in the canonical ensemble with fixed particle number and volume (constant-NVT). The EOM for each particle is integrated with the velocity-Verlet [21] algorithm with a time step $\Delta t=0.006 \tau$, where $\tau=\sigma(m / \epsilon)^{1 / 2}$ fixes the time scale. We set $\Gamma=0.5 \tau^{-1}$. All results presented here are measured in reduced units, as derived from the fundamental scales fixed by $\sigma, \epsilon, m$, and the Boltzmann constant $k_{B}$. To reduce computation time we use a combination of the Verlet and linked-cell list algorithms [21].

Periodic boundary conditions are used in all three space dimensions, thus forcing the creation of (at least) two interfaces in a two-phase system. The system sizes, temperatures, particle numbers and equilibration times of our simulations are listed in Table I. In Table I and throughout this paper, $L$ refers to the dimensions of the square cross section parallel to the interface, which lies in the $x y$ plane. Thus, $L_{x}=L_{y}=L_{\|}=L . L_{\perp}$ refers to the dimension of the box perpendicular to the plane of the interface. Simulations are performed for $L$ ranging from $12.8 \sigma$ to $134.6 \sigma$. The largest system we could run

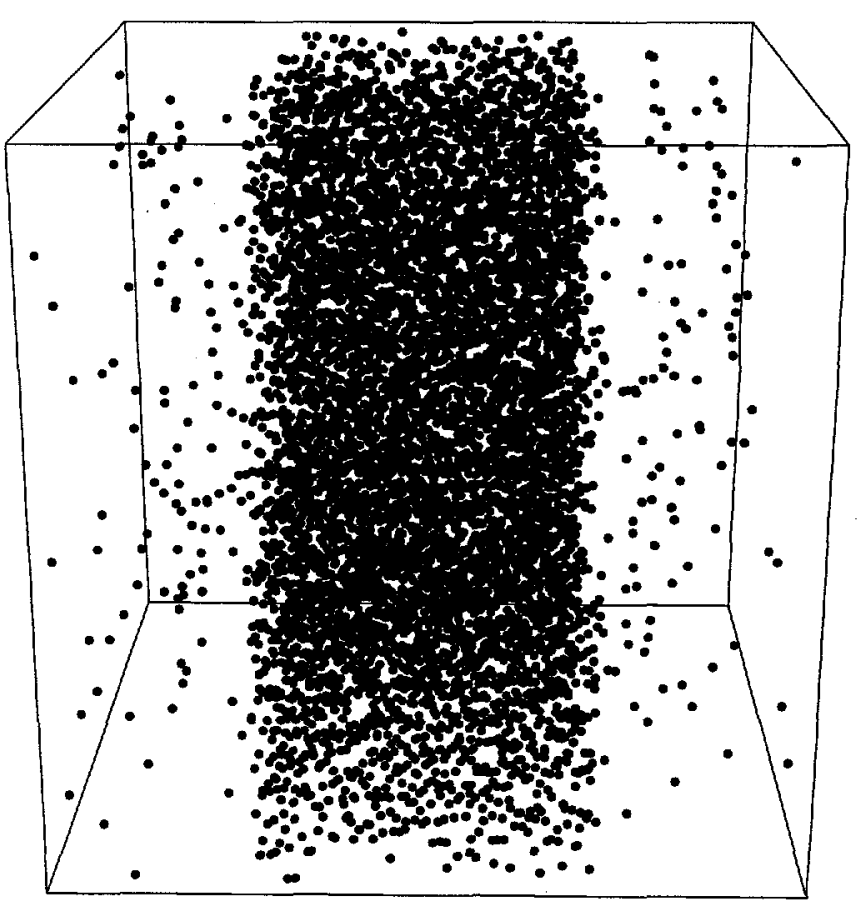

FIG. 1. Typical configuration of an equilibrated liquid/vapor interface at $T=0.8 \mathrm{\epsilon} / k_{\mathrm{B}}$. Length of square cross section holding the interface is $L=12.8 \sigma$.

\begin{tabular}{c||cccc}
\hline \hline$T\left(\epsilon / k_{\mathrm{B}}\right)$ & $\rho_{V}$ & $\rho_{L}$ & $\gamma_{w_{e}}$ & $\gamma_{p}$ \\
\hline \hline 0.8 & $0.020(1)$ & $0.730(1)$ & $0.37(3)$ & $0.39(1)$ \\
\hline 0.9 & $0.045(1)$ & $0.663(1)$ & $0.22(1)$ & $0.22(1)$ \\
\hline 0.95 & $0.066(1)$ & $0.623(1)$ & - & $0.15(1)$ \\
\hline 1.0 & $0.098(2)$ & $0.571(1)$ & $0.097(2)$ & $0.08(1)$ \\
\hline \hline
\end{tabular}

TABLE II. Calculated values of the bulk densities and surface tensions for different simulation temperatures.

contains 1.24 million particles. After that, computation becomes prohibitively slow due to the large number of particles. At the other end of our size range, systems with $L<12 \sigma$ demonstrate non-negligible finite-size effects. Figure 1 shows a typical configuration of an equilibrated system of $L=12.8 \sigma$ at $T=0.8 \epsilon / k_{\mathrm{B}}$.

Initial systems were built as follows: for each system size and temperature, we construct a slab of the liquid phase and center it in the middle of the simulation cell with the interface perpendicular to the $z$ direction. $L_{\perp}$ is set such that it is at least twice the length of the liquid slab, allowing sufficient space for the bulk liquid and vapor densities to achieve constant values. Since the phase coexistence diagram is well known for this system $[17,18,22]$, we adjusted the density of the liquid and vapor regions to be close to their reported values for each temperature $T$. 


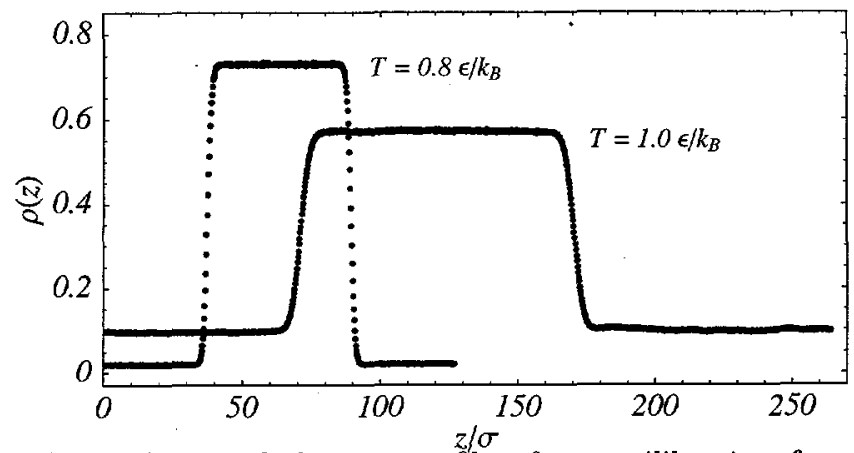

FIG. 2. Averaged density profile after equilibration for $T=0.8$ and $1.0 \epsilon / k_{\mathrm{B}}$ for $L=41.9 \sigma$.

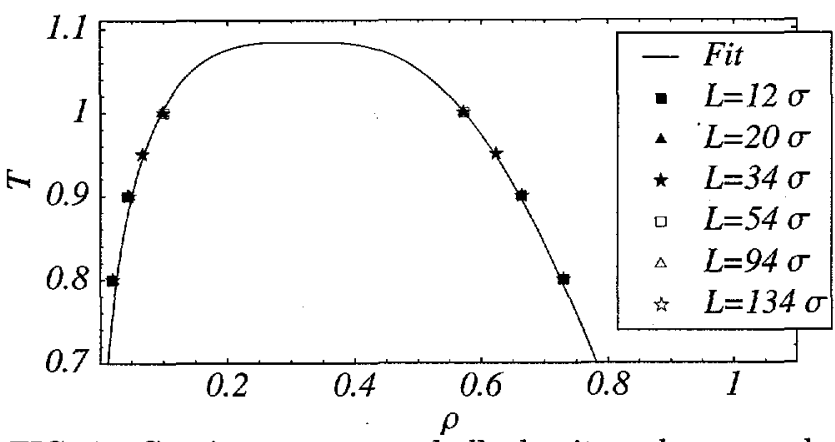

FIG. 3. Coexistence curve: bulk density values are obtained from tail values of interfacial liquid/vapor density profiles. Curve is a best fit using the following expressions [18], $0.5\left(\rho_{L}+\rho v\right) \sigma^{3}=0.544-0.210 k_{B} T / \epsilon$ and $\left(\rho_{L}-\rho_{V}\right) \sigma^{3}=A\left(1-\left(T / T_{c}\right)\right)^{0.318}$. The best fit parameters are $A=1.07$ and $T_{c}=1.085$.

After the system has equilibrated the density profile is measured, i.e. the $x y$-cross-section averaged number density $\rho(z)$ as a function of $z$. Over the course of a simulation for a given $T$ and $L, \rho(z)$ is measured every 400 time steps. Once the interfaces have equilibrated, the density profiles are averaged over $10^{5}-2 \times 10^{5} \Delta t$. Great care must be exercised in the averaging procedure. For each profile, the position of the interface is located to insure that the averaging does not artificially broaden the interface width due to drift in the interface positions. Figure 2 shows an example of an equilibrated, averaged density profile for $T=0.8$ and $1.0 \epsilon / k_{\mathrm{B}}$ for $L=41.9 \sigma$.

Bulk values for the density are extracted from the tail values (obtained through a fit described below) of the interfacial density profiles. Our final equilibrated values for the bulk liquid and vapor densities are listed in Table II, and agree very well with values reported in the literature. The derived coexistence curve, along with a fit to an expression suggested in Ref. [18] are shown in Fig. 3. The very good agreement of the bulk values suggests that our systems are well equilibrated that our measurement procedures are sound. Since the simulations are started near their respective liquid and vapor values, the bulk density values shown in Fig. 3 all attained equilibrium values quickly. However, the interface structure did not equilibrate until the simulations had been run for the much longer times shown in Table I.

An important quantity characterizing the interface is the width. The intrinsic width of an interface is due to the intermixing of the two phases, which always occurs to a certain degree at finite, subcritical temperatures. In addition to this mixing, capillary-wave theory [4] predicts that thermal fluctuations of the location of the interface will contribute to the total, cross-section averaged, measured width. This broadening depends primarily on the surface tension, the temperature, and the cross-sectional size of the interface, and the spatial dimension. As an example, capillary-wave theory states that any two-dimensional crystal is unstable against thermal fluctuations [23].

Fluctuations in $\zeta(x, y)$, the mean location of the interface in the $z$ direction, induces fluctuations in the total area of the interface and can be easily determined by expanding the shape of the interface to first order. This approximation is accurate provided the interface is smooth, with no overhangs. The free energy of the interface is the product of its surface area and an interfacial energy density $\gamma$, which is assumed to be independent of local curvature. Fluctuations due to capillary waves have an energy cost due to the increase in the surface area of the interface. The resulting interfacial Hamiltonian can be expressed as the product of surface tension times the increase in interfacial area

$$
\begin{aligned}
\mathcal{H}\{\zeta\} & =\gamma \int d x d y\left[\sqrt{1+\frac{\partial \zeta}{\partial x}} \sqrt{1+\frac{\partial \zeta}{\partial y}}-1\right] \\
& \approx \frac{\gamma}{2} \int d x d y|\nabla \zeta(x, y)|^{2}
\end{aligned}
$$

The capillary-wave spectrum can be calculated by substituting the Fourier transform of $\zeta$ giving

$$
\mathcal{H}\{\zeta\} \approx \frac{\gamma}{2} \int d \vec{q} q^{2}|\tilde{\zeta}(\vec{q})|^{2}
$$

where $\vec{q}$ represents a two-dimensional vector in reciprocal space, and $\mathcal{F}[\zeta(x, y)]=\tilde{\zeta}(\vec{q})$ is the Fourier transform of $\zeta(x, y)$. The equipartition theorem dictates the meansquare amplitude for each interfacial excitation mode,

$$
\left\langle|\tilde{\zeta}(\vec{q})|^{2}\right\rangle=\frac{k_{\mathrm{B}} T}{4 \pi^{2} \gamma q^{2}}
$$

and summing over all allowed modes, one gets

$$
\begin{aligned}
\left\langle|\tilde{\zeta}|^{2}\right\rangle & =\frac{k_{\mathrm{B}} T}{4 \pi^{2} \gamma} \int_{q_{\min }}^{q_{\max }} \frac{d \vec{q}}{q^{2}}, \\
& =\frac{k_{\mathrm{B}} T}{2 \pi \gamma} \ln \left(\frac{L}{B_{\mathrm{o}}}\right),
\end{aligned}
$$

where $q_{\min }=2 \pi / L$ and $q_{\max }=2 \pi / B_{o}$ Note that both lower and upper cut-offs are required to prevent the value of 
the integral from diverging. The long-wavelength cutoff $q_{\min }$, is determined by $L_{\|}$[24]. The interpretation of the short-wavelength cutoff, $q_{\max }$, is not as clear. Werner et al. $[14,15]$ have studied the dependence of $B_{o}$ for polymer/polymer interfaces and suggest $B_{o}$ scales inversely with the molecular weight. However, the exact nature of this short-wavelength cut-off remains an open question.

In both simulations and experiments, the quantity measured is the total interfacial width, which includes contributions from the intrinsic width and the broadening due to capillary-wave fluctuations. The two effects can be distinguished if one assumes that capillarywave fluctuations are decoupled from the intrinsic profile. Therefore, the total interface profile $\Psi(z)$ may be expressed as a convolution of the intrinsic interface profile $\psi(z)$ and the effect due to capillary waves [16],

$$
\Psi(z)=\int_{-\infty}^{\infty} \psi\left(z-z_{o}\right) P\left(z_{o}\right) d z_{o} .
$$

Here, $P\left(z_{o}\right)$ is the probability of finding the interface at $z_{o}$, i.e.,

$$
P\left(z_{o}\right)=\frac{1}{L_{x} L_{y}} \int_{0}^{L_{x}} \int_{0}^{L_{y}} d x d y \delta\left(\zeta(x, y)-z_{o}\right) .
$$

The interfacial order parameter profile $\Psi(z)$ is related to the cross-section averaged density profile $\rho(z)$ by the function

$$
\Psi(z)=\frac{2}{\rho_{L}-\rho_{V}}\left[\rho(z)-\frac{\rho_{L}+\rho_{V}}{2}\right],
$$

which scales the density profile so that $\Psi(z)$ varies between -1 and 1 . The variance of the derivative of the total profile $d \Psi(z) / d z \equiv \Psi^{\prime}$ can be used as a measure of the width of the interface. The variance of a distribution $f$ is given by

$$
v[f]=\frac{\int_{-\infty}^{\infty} z^{2} f(z) d z}{\int_{-\infty}^{\infty} f(z) d z}=\frac{-\left.\frac{d^{2}}{d q^{2}} \tilde{f}(q)\right|_{q=0}}{\tilde{f}(0)}
$$

where $\tilde{f}(q)$ is the Fourier transform of $f(z)$. Making use of the convolution theorem and Eqs. (9) and (12) it can be shown that [16]

$$
\begin{aligned}
v\left[\Psi^{\prime}\right] & =v\left[\psi^{\prime}\right]+v[P], \\
\Delta^{2} & =\Delta_{o}^{2}+\frac{k_{\mathrm{B}} T}{2 \pi \gamma} \ln \left(\frac{L}{B_{o}}\right) .
\end{aligned}
$$

The squared widths of the total and intrinsic interfacial profiles have been defined as $\Delta^{2} \equiv v\left[\Psi^{\prime}\right]$ and $\Delta_{o}^{2} \equiv v\left[\psi^{\prime}\right]$, respectively. Note that the average squared fluctuations of the interface about its mean location in the $z$ direction can be directly identified as $\left\langle|\zeta|^{2}\right\rangle=v[P]$. Thus, our choice of measure for the interfacial width clearly shows that the total interfacial width can be written as the sum of an intrinsic part and a contribution due to capillarywave fluctuations.

In order to verify this prediction, we performed several simulations on different system sizes. Traditionally, the order parameter interfacial profile has been fit with $f(z)=\tanh \left(2 z / w_{t}\right)$ or an error function $\operatorname{erf}\left(\sqrt{\pi} z / w_{e}\right)$. Using our data we can test these two fitting functions and the resulting predictions for $\gamma$. Another reason for fitting our results for $\Psi(z)$ to one of these two functions is that we found we can determine a value for $\Delta^{2}$ more accurately than by extracting it directly from the data; once the fitting parameters of $f(z)$ have been determined, $v[f]$ can be easily calculated. The two different fitting functions we tested are

$$
\begin{aligned}
& f_{e}\left(z, w_{e}\right)=\operatorname{erf}\left(\frac{\sqrt{\pi} z}{w_{e}}\right), \\
& f_{t}\left(z, w_{t}\right)=\tanh \left(\frac{2 z}{w_{t}}\right) .
\end{aligned}
$$

For these two functions, the variance of each in terms of their associated widths $w_{x}$ 's are

$$
\Delta^{2} \rightarrow\left\{\begin{array}{l}
v\left[f_{e}^{\prime}\right]=w_{e}^{2} / 2 \pi \\
v\left[f_{t}^{\prime}\right]=\pi^{2} w_{t}^{2} / 48
\end{array}\right.
$$

The simulations are performed for three temperatures, $T=0.8,0.9$, and $1.0 \epsilon / k_{\mathrm{B}}$. This range of temperatures is selected because at lower temperatures the interfacial width is comparable to the average interparticle distance, and therefore is difficult to measure accurately. The upper bound is set by $T_{c} \approx 1.085 \epsilon / k_{\mathrm{B}}$. For each value of $T$, the profiles are fit to both $f_{x}$ 's described above. Near the interface, the fitting functions can hardly be distinguished. In fact, some studies have used an error function for theoretical derivations while using a hyperbolic tangent function to fit their data $[13,19]$.

We fit our interfacial profiles for data near the interface and data deep into the bulk liquid and vapor regions. There is no a priori requirement that a liquid/vapor density profile must be symmetric about the center of the interface. However, we detected no significant amounts of asymmetry. For each temperature and system size, the simulations are run until the interfacial profiles show a constant $\Delta^{2}$.

Figure 5 summarizes the analysis from our extensive molecular dynamics simulations of a liquid/vapor interface. For both tanh and erf fits, the data confirm a logarithmic dependence of $\Delta^{2}$ on system size. Using Eq. (13) the surface tensions can be calculated from the slopes of the best fit lines in Fig. $5\left[\gamma_{w}=k_{\mathrm{B}} T /(2 \pi\right.$ slope $\left.)\right]$. The temperature dependence of the interfacial surface tensions calculated from our simulations are shown in Fig. 4. We compare these values of the surface tension with an independent measurement obtained from the components of the pressure tensor, $\gamma_{p}=L_{\perp}\left(p_{\perp}-p_{\|}\right)$[25], represented 


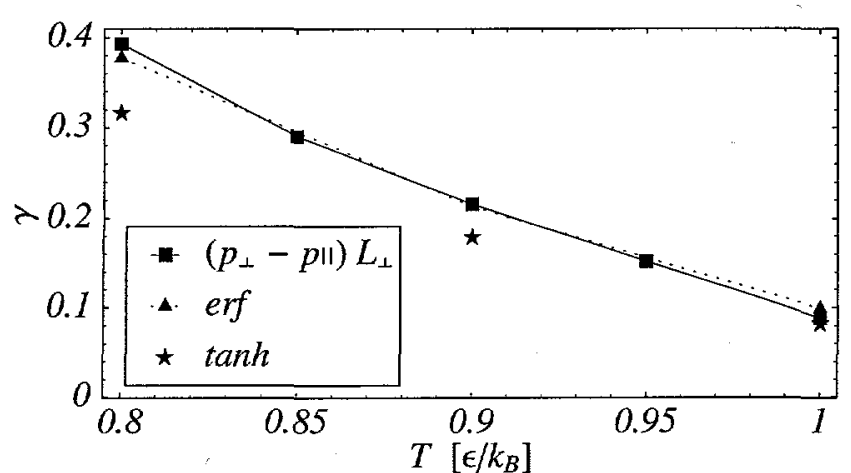

FIG. 4. Surface tension vs temperature $T$. Results for the surface tension $\gamma_{w}$ obtained from fitting interfacial profiles with a tanh or erf are compared to the values calculated from the components of the pressure tensor, $\gamma_{p}=L_{\perp}\left(p_{\perp}-p_{\|}\right)$. The error function results are in excellent agreement with $\gamma_{p}$ while the tanh results are systematically $15 \%$ too low: The $\gamma_{p}$ value for $T=0.85$ is taken from Ref. [18]. The $\gamma_{p}$ value for $T=0.95$ is calculated from a single simulation with $L=41.9 \sigma$.

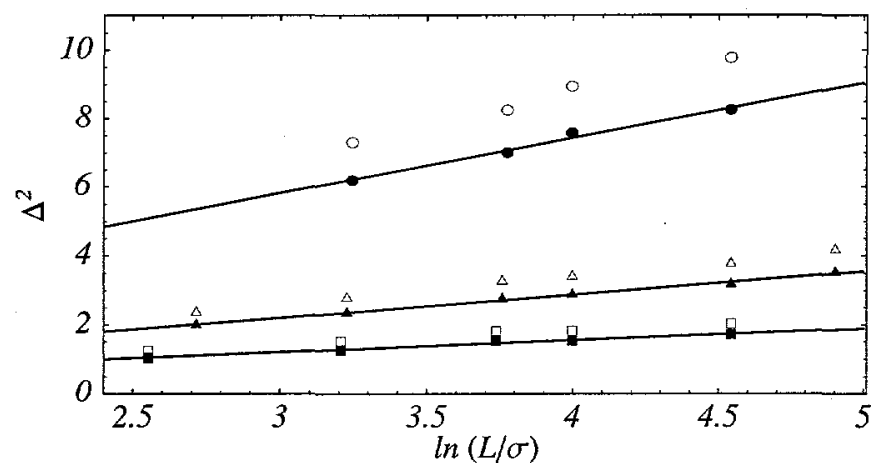

FIG. 5. Variance $\Delta^{2}$ versus $\ln L$ for $T=0.8$ (squares), 0.9 (triangles) and $1.0 \epsilon / k_{\mathrm{B}}$ (circles). The open and solid symbols are obtained using hyperbolic tangent and error function fits to the interfacial profiles, respectively. Lines are linear least-squares fits to the error function data.

by solid squares. The agreement between $\gamma_{p}$ and $\gamma_{w}$ obtained from the error function fits is very good. Using the tanh fits we obtain surface tensions that are systematically $15 \%$ lower than those from the error function fits, which follows from their larger slopes shown in Fig. 5. Thus we obtain the somewhat unsettling result that the value of the $\Delta^{2}$ and hence $\gamma_{w}$ depends on the form of the fitting function used to fit $\psi(z)$.

To investigate the systematic discrepancy between the tanh and erf fits, we performed an unweighted fit a hyperbolic tangent function to data generated with an error function. The results are shown in Fig. 6 . The integrand in the numerator of the variance is plotted vs $z$ for both functions. The integral of each of these functions is proportional to $\Delta^{2}$. From Fig. 6 one can see that the tails in the integrand of the tanh function contribute more significantly than the error function, hence the larger measured values of $\Delta^{2}$ from the tanh fits. We conclude that the tails of interfacial profiles are better captured by fits
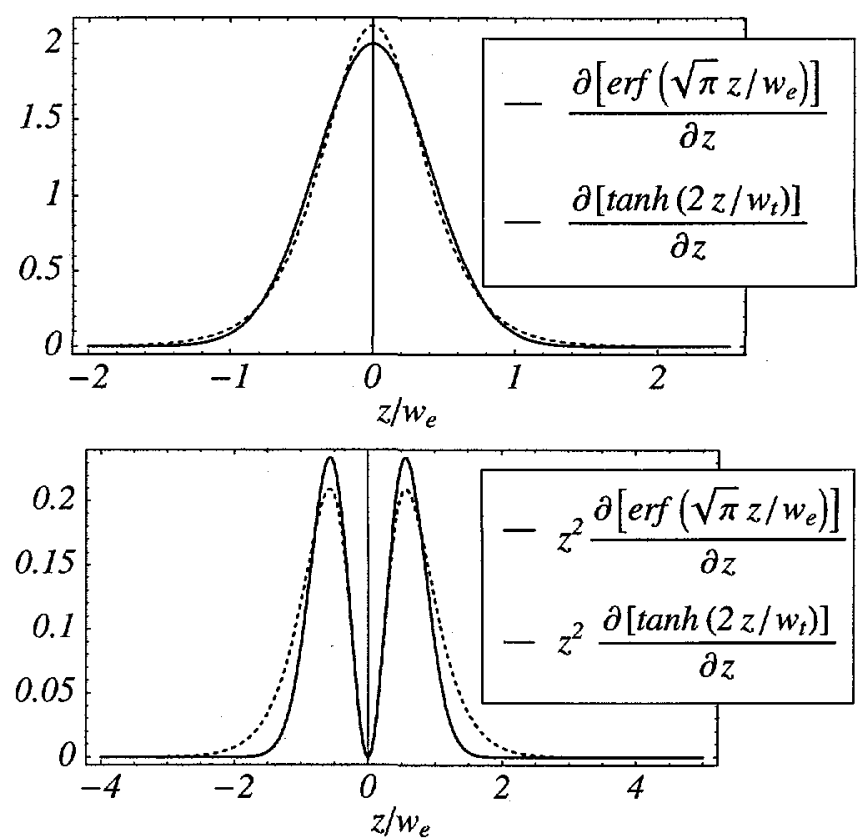

FIG. 6. Results for an unweighted fit of a tanh function to a error function for $w_{e}=1$.

to an error function.

In this paper, we presented results of extensive molecular dynamics simulations of liquid/vapor interfaces. Our data confirm the capillary-wave description of the interface structure between a Lennard-Jones liquid and its vapor phase. When measuring the interfacial width by using second moments of the interfacial profile derivatives, we can extract values for the surface tension that agree very well with calculations from the components of the pressure tensor. We have also shown that the more robust method of extracting the second moment is through fits to an error function, since using a hyperbolic tangent leads to systematic errors.

The results presented here are for an isolated liquid/vapor interface. The width of the liquid and vapor regions were carefully chosen so that there was no interference between the two interfaces. An interesting extension of this work is to study the effect of a nearby substrate on an interface. The effect of a wall on the interface can be modeled by adding a potential energy term to the interface Hamiltonian, $\mathcal{H}[d]$, which depends on the distance $d$ between the interface and the wall. This term is calculated by integrating the potential energy between the microscopic constituents of two macroscopic objects, i.e. the interface and a semi-infinite wall. For pure LJ interactions, the potential energy is proportional to $A / d^{2}$, where $A$ is the Hamaker constant [26]. The Hamaker constant contains information about the strength of the microscopic potential, geometrical factors, and macroscopic properties of the wall. Since this additional term in the Hamiltonian is quadratic in $d, \mathcal{H}$ can therefore be diagonalized by a Fourier transform and the deriva- 
tion of the capillary-wave spectrum is similar to the one presented here. The effect of the substrate is to cut off the long wavelength capillary-wave fluctuations so that $\Delta^{2}$ no longer depends on $L_{\|}$for small $d$. The interplay between $L_{\|}$and $d$ is an interesting question for which computer simulations such as these can directly address.

We thank Frank van Swol for helpful discussions. Sandia is a multiprogram laboratory operated by Sandia Corporation, a Lockheed Martin Company, for the United States Department of Energy under Contract DE-AC0494AL85000.

[1] R. Evans, R. L. de Carvalho, J. Henderson, and D. Hoyle, J. Chem. Phys. 100, 591 (1994).

[2] S. Rice, Non-Cryst. Solids 205-207, 755 (1996).

[3] D. Chekmarev, M. Zhao, and S. Rice, J. Chem. Phys. 109, 768 (1998).

[4] F. Buff, R. Lovett, and F. Stillinger, Phys. Rev. Lett. 15, 621 (1965).

[5] J. Rowlinson and B. Widom, Molecular Theory of Capillarity (Clarendon Press, Oxford, 1982).

[6] M. Sanyal, S. Sinha, K. Huang, and B. Ocko, Phys. Rev. Lett. 66, 628 (1991).

[7] I. Tidswell, T. Rabedeau, P. Pershan, and S. Kosowsky, Phys. Rev. Lett. 66, 2108 (1991).

[8] M. Tolan et al., Phys. Rev. Lett. 81, 2731 (1998).

[9] K. R. Shull, A. M. Mayes, and T. P. Russell, Macromolecules 26, 3929 (1993).

[10] M. Sferrazza et al., Phys. Rev. Lett. 78, 3693 (1997).

[11] J. Scherble et al., Macromolecules 32, 1859 (1999).

[12] T. Kerle, J. Klein, and K. Binder, Phys. Rev. Lett. 77, 1318 (1996).

[13] A. Werner, F. Schmid, M. Müller, and K. Binder, J. Chem. Phys. 107, 8175 (1997).

[14] A. Werner, F. Schmid, M. Müller, and K. Binder, Phys. Rev. A 59, 728 (1999).

[15] A. Werner, M. Müller, F. Schmid, and K. Binder, J. Chem. Phys. 110, 1221 (1999).

[16] M.-D. Lacasse, G. S. Grest, and A. J. Levine, Phys. Rev. Lett. 80, 309 (1998).

[17] M. Nijmeijer, A. Bakker, C. Bruin, and J. Sikkenk, J. Chem. Phys. 89, 3789 (1988), and references therein.

[18] P. Adams and J. Henderson, Molecular Physics 73, 1383 (1991).

[19] T. Hapke, G. Pätzold, and D. W. Heermann, J. Chem. Phys. 109, 10075 (1998).

[20] M. Doi and S. Edwards, The Theory of Polymer Dynamics (Clarendon Press, Oxford, 1986).

[21] M. Allen and D. Tildesley, Computer Simulation of Liquids (Clarendon, Oxford, 1987).

[22] B. Smit, J. Chem. Phys. 96, 8639 (1992).

[23] K. Huang, Statistical Mechanics (John Wiley, New York, 1987).

[24] For systems in a gravitational field $g$, a gravitational length defined by $l_{g}^{2}=2 \gamma / g \Delta \rho$ determines the upper cut- off, where $\Delta \rho$ is the difference in bulk density between the two phases.

[25] T. Hill, An Introduction to Statistical Thermodynamics (Dover, New York, 1986)

[26] J. Israelachvili, Intermolecular \& Surface Forces (Academic Press, London, 1991). 